\title{
Research on Tunnel Deformation Monitoring based on BIM Technology
}

\author{
Zhizhang Wan, Ping Gan \\ Nanchang Institute of Science \& Technology, Nanchang, Jiangxi, China, 330108
}

Keywords: BIM, Tunnel Deformation Monitoring, Engineering Development

\begin{abstract}
BIM technology is the construction industry in the application of more and more widely, but also to promote the development of the construction industry. BIM technology with high efficiency, high quality and other characteristics, so that the construction of Tunnels to overcome the complexity and reduce the difficulty of the project. In this paper, the BIM technology in the Tunnel design problems, introduced the relevant application methods, hoping to improve the quality of Tunnel design, and promote the development of BIM technology.
\end{abstract}

\section{Introduction}

In recent years, the use of BIM technology in architecture, machinery and electronics has matured and has made innovative changes, while the informationization of the railway industry and the utilization of BIM technology are still at a low level. With the development of China's railway industry, the Tunnel project is paying more and more attention to the control of investment, the design rigor, the safety and operation of construction, and gradually turn to low carbon, environmental protection and sustainable development. At the same time, with the increasingly fierce competition and challenge of domestic and foreign industry, BIM and three-dimensional design technology effectively combined and applied to the Tunnel design stage, in order to improve the core competitiveness of the design unit is an inevitable direction of development.

At present, the railway industry three-dimensional design and BIM technology applications are in the exploratory stage, so the new design concept to be put forward, effective design tools to be studied, the traditional design process to be innovative. Based on the deep research on the theory of BIM, this paper puts forward the technical route of applying BIM technology to the three-dimensional design of Tunnel, and puts forward the practical application in combination with the high risk tunnel project of Mountain in order to further improve our country Tunnel BIM technology application level to contribute.

Based on the BIM concept pioneered by Professor Chuck Eisman, the definition of BIM in the relevant national standards, BIM in Tunnels can be defined as "the process of planning, designing, constructing and operating the Tunnel project using the information model The model contains information on the geometrical, spatial, structural, and performance aspects of the project, and the project participants should maintain the model together and work on the basis of relevant information. The three-dimensional design of the Tunnel is to carry out the three-dimensional line selection on the basis of the establishment of the three-dimensional geological model, and carry out the three-dimensional design work of the tunnel. The main contents of the work include sketch design, 3D modeling, information addition, engineering statistics and engineering Etc., should have a higher collaborative design and resource management performance.

\section{BIM Technology Features}

The integration of IBM technology is generated through a three-dimensional model database. In terms of engineering implementation, the application of IBM technology can integrate the different phases of the project together to achieve integrated management of the project, which can be translated into a three-dimensional model by integrating and integrating information The Relative to the two-dimensional form, the application of IBM technology to data information becomes more intuitive and specific. 
Compared with other projects, the subway tunnel project has more obvious linear characteristics. under these circumstances. If the entire subway tunnel project is built into an IBM model, the required workload is relatively large, and the modeling is relatively difficult. Therefore, the entire subway tunnel project can be divided into several different parts according to the geological conditions and other factors, and an IBM model is built in each part. Once all of the part of the modeling work is completed, these IBM models can be in accordance with the geographical location, Metro line mileage parameters such as reasonable integration together. In order to give full play to the visualization of IBM technology, Simulation advantages, in the actual subway tunnel construction process, you can use IBM technology to build a subway tunnel engineering IBM management platform. In order to ensure the application quality of IBM management platform, IBM management platform can be designed by the following elements: application layer. This element directly is to the subway tunnel project to produce the corresponding role. Such as the construction of materials for the subway tunnel project construction equipment and other resources to carry out management to carry out 26 visualization of the end of the management work, 26 construction optimization simulation work. This element is the middle tier of the entire Metro tunnel IBM management platform, which is geared towards both the data layer and the application layer. In the IBM management platform running process, the model layer from the application layer to obtain the appropriate data information, such as subway tunnel construction progress, Construction quality, Engineering parameters and so on. And then use their own integrated function to collect the data obtained by the data transfer to the IBM management platform data layer, Data layer. In the IBM management platform, the data layer function mainly refers to: data format conversion function. When the non-(78 format data) is obtained from the model layer, the data layer can use the data conversion interface to convert the above format data into the IFC format and supply the IBM management platform for data analysis.

\section{BIM Technology in the Three-Dimensional Design and Application of Technical Routes}

BIM technology is not the application of a single software platform, but the cooperation of multiple software, and requires the information model can be in the software between the lossless exchange, seamless link, so you can choose the same series of software to achieve BIM technology applications The This article from the software performance, professional requirements, hardware and software environment and other aspects of the series of software at home and abroad for a comprehensive comparison, the final selection of Dassault series of software as a technical support, outlined as follows:

(1) ENOVIA: collaborative management platform, responsible for the entire life cycle of information coordination and data management;

(2) CATIA: product design platform for the design phase to establish three-dimensional model;

(3) DELMIA: simulation application platform for the construction of the dynamic simulation and construction plans for the production;

(4) 3DVIA: three-dimensional display platform for the design of lightweight models such as display. In this paper, the concept of skeleton design is applied to the three-dimensional design of the tunnel, in order to facilitate the collaboration between the professional and professional. At the same time, parametric modeling, template encapsulation, directory call and other functions used in three-dimensional modeling, greatly improving the efficiency and accuracy of modeling.

\section{Problems in Tunnel Monitoring Design}

The Tunnel project is a project with complexity and particularity. Tunnel design involves a lot of complex knowledge, the need for design planners in the railway expertise to be rich enough and can be skilled use. In addition to the need to master a lot of edge knowledge, the knowledge is often used when the design needs. (2) At present, the common drawings in China's Tunnel design are two-dimensional drawings, as a basis for delivery, but the two-dimensional drawings of this delivery basis in the embodiment of three-dimensional solid project has a limit, coupled with the 
construction of the Tunnel construction Personnel if there is an error, misunderstanding of the meaning contained in the design drawings, the final direct impact on the construction of a reasonable, reduce the quality of the project. (3) Compared with the construction industry, the Tunnel has more complicated characteristics, and can not only rely on our traditional design method to complete the Tunnel design and construction.

BIM technology in the Tunnel application will have a profound impact on the development of the railway industry. BIM technology in the Tunnel industry can not only the contents of the design drawings for detailed analysis, but also according to the actual situation of the construction area, optimize the design. In addition, when the various parts of the construction of the Tunnel organically combined, the use of BIM technology will make each component between the formation of a very meaningful link, the Tunnel design can be more carefully examined, so as to Reduce the error rate in the design. Designers in the use of BIM technology, the Tunnel design will involve the entity to reduce the calculation of the contents of the BIM technology can be used to reduce the error generated by man-made calculations. BIM technology can not only change the amount of engineering changes, but also has the role of visual display, the same can reduce the cost of the project to reduce the emergence of errors.

Currently there are BIM technology software in the international arena, according to international standards can be divided into two categories, one is to create BIM model of software, this category includes the core modeling software, program design software, as well as interface geometry Modeling software; the second is the use of BIM model software, which mainly has sustainable green analysis software, structural analysis software, cost management software. In order to consider the quality of the Tunnel project, in general, the design planners will try to choose the series and compatibility are better performance of the software, the purpose is to ensure the late application of the exchange process data integrity, but the premise is the design planners To the advantages of various software to coordinate with the Tunnels for the construction of the program. General use of BIM technology in China's Tunnel design, will consider the use of CATIAV5 software as the core modeling software. This modeling of the software in the Tunnel design will make BIM design more systematic, and also conducive to BIM technology to play their own advantages.

\section{Tunnel Deformation Monitoring}

The traditional method of monitoring the deformation of the tunnel is compiled into a monitoring report by means of data tables, two-dimensional deformation curves and text description. The project participants discuss the monitoring results, analyze whether the deformation is too large or whether it is stable, Timely feedback and analysis, and determine whether to take the necessary remedial, rescue measures, so that the tunnel does not occur accidental damage and deformation. Every day by manual operation, read a large number of Excel table pages of thousands of data, each measured point of the deformation and cumulative deformation value, to determine the risk of critical state. If you want to know the deformation rate, it can only be a small number of sensitive points one by one to describe its single point of time deformation trajectory, to analyze the trend, and then be marked with the alarm. There are several major defects, the first time and effort, is not conducive to the rapid deformation of the tunnel to determine; the second is to rely on large artificial data to read, easy to ignore the leak; the third can not visit the tunnel section of the massive lateral deformation and The three-dimensional relationship between the vertical vertical settlement of the monitored pipeline; the fourth can not visually see the deformation time trend of the whole tunnel and quickly find the hazard source.

The 5M foundation pit deformation monitoring technology is used to expand the BIM model which has been established in the design stage, so that the deformation monitoring of the foundation pit is more convenient and fast, and the deformation sensitive point and the dangerous point can be extracted accurately and quickly. Can be found in the first time the risk of deformation of the foundation pit and the degree of danger, whether to start contingency plans and contingency plans have a great help, and can make the pit monitoring and management personnel in the complex read 
the report of the work Liberated. Based on BIM, the application of monitoring technology of foundation pit 5D makes the safety monitoring of foundation pit construction rely on visual means to improve the working rate of foundation pit monitoring and effectively reduce the man-made omission in safety monitoring process. BIM technology in Baoli building foundation pit safety monitoring to expand the application of exploration of the pit pit safety monitoring of the new method for the BIM technology in the construction of the whole process of foundation pit provides a practical reference.

\section{Conclusion}

The construction of the Tunnel requires the construction of qualified personnel, and its design work may introduce professional and technical. The application of BIM technology in Tunnels is a general trend, which is beneficial to the improvement of the quality of Tunnels in China and the improvement of the quality of Tunnels. In the future need to continue efforts to promote the application of BIM technology and the project construction have a leap-style impact, improve the quality of Tunnel design.

\section{References}

[1] Meng Haobo. Municipal Corridor Foundation Pit Construction Monitoring Analysis [J]. Urban Road Bridge and Flood Control, 2017 (05)

[2] Lin Yanshan. Comparative analysis of deformation characteristics of foundation pit in coastal areas and eastern inland areas of southern Guangdong [J]. Construction Supervision and Testing and Cost, 2017 (01)

[3] Zhao Shaomin, Ji Hongqi. Analysis of the application of foundation pit detection technology [J]. Building materials and decoration, 2017 (14)

[4] Xie Haicheng. Common problems and countermeasures of construction pit monitoring [J]. Building materials and decoration, 2017 (16)

[5] Yang Yutao, Zhou Yi, Zhang Lei. Application of Foundation Pit Support and Precipitation Engineering Technology [J]. Construction Technology Development, 2017 (02) 